\title{
Leakage of Potassium from Red Blood Cells following Gamma Ray Irradiation in the Presence of Dipyridamole, Trolox, Human Plasma or Mannitol
}

\author{
Junichi Hirayama, * Hideki Abe, Hiroshi Azuma, and Hisami Ikeda \\ Hokkaido Red Cross Blood Center; 2-2 Yamanote, Nishi-ku, Sapporo 063-0002, Japan. \\ Received January 17, 2005; accepted March 23, 2005
}

\begin{abstract}
Transfusion-associated graft-versus-host disease (TA-GVHD) is a fatal complication of blood transfusion resulting from the contamination of blood products by leukocytes. In order to prevent this disease, gamma or Xray irradiation of blood components, which can inactivate leukocytes, is currently used. However, the minimal doses needed to destroy lymphocytes promote the leakage of potassium from red blood cells (RBCs), which can induce other side effects, such as hyperpotassemia and cardiac arrest. The reactive oxygen species (ROS) generated by the irradiation of aqueous solutions may accelerate the leakage through oxidation of the RBC membrane. Here we studied the effect of dipyridamole, Trolox, human plasma or mannitol on the leakage of potassium from RBCs following irradiation. RBC preparations (hematocrit; 30\%) containing antioxidants were irradiated at $30 \mathrm{~Gy}$ and stored at $4{ }^{\circ} \mathrm{C}$ for $7 \mathrm{~d}$. The leakage of potassium from the RBCs caused by the irradiation was significantly suppressed by dipyridamole (more than $50 \mu \mathrm{M}$ ), Trolox (more than $5 \mathrm{~mm}$ ) or human plasma (50\%). Mannitol (80 $\mathrm{mm})$ is used to inhibit hemolysis as a constituent of MAP solution, which is a solution used for the storage of RBC products in Japan. Here it was clarified that the leakage of potassium from not only irradiated but also non-irradiated RBCs was unexpectedly promoted by mannitol. The amount of mannitol in MAP solution may have to be reconsidered. The osmotic pressure of the RBC preparation increased in a manner dependent on the concentration of mannitol. The elevated osmotic pressure may promote the leakage. In conclusion, although antioxidants have the potential to suppress the leakage of potassium ascribed to the irradiation, the extent of the supression (10-20\%) by dipyridamole (DPM), Trolox or human plasma seems insufficient for the clinical use of these agents as an additive for MAP solution.
\end{abstract}

Key words dipyridamole; irradiation; mannitol; red blood cell; Trolox; potassium

Transfusion-associated graft-versus-host disease (TAGVHD) is a complication of blood transfusion that results from the engraftment and clonal expansion of allogenic donor white cells. ${ }^{1)}$ Although TA-GVHD is rare, patients respond poorly to treatment and the disorder is usually fatal. ${ }^{2)}$ Radiation treatment of blood components is currently used to prevent TA-GVHD. ${ }^{3)}$ This procedure is based on the large difference between the radiation sensitivities of T-lymphocytes and red blood cells (RBCs). ${ }^{3,4)}$ Thus, one has to apply a dose high enough to destroy almost all the T-lymphocytes while causing as little damage as possible to RBCs. Recent studies indicate that the minimal dose required to fulfill this requirement is from 15 to $50 \mathrm{~Gy}^{5}{ }^{5,6)}$ Doses of this order of magnitude cause only minor changes in the essential constituents of RBCs, e.g. intracellular hemoglobin, ATP, membrane lipid and proteins. However, they promote the leakage of potassium from RBCs. ${ }^{78)}$ There have been concerns about possible side effects, such as hyperpotassemia and cardiac arrest, of the transfusion of blood components with an elevated potassium concentration. ${ }^{9-11)}$

The irradiation of aqueous solutions can lead to the generation of reactive oxygen species (ROS) such as the hydroxyl radical which is a powerful oxidant and can interact with lipids and proteins in the membranes of RBCs. ${ }^{12)}$ It was reported that the increase in the extracellular potassium concentrations of RBC preparation on photosensitization resulted from the oxidative damage of RBC membranes by ROS produced by photosensitization, and furthermore, that the increase was sufficiently inhibited by the addition of an antioxidant such as dipyridamole (DPM) or Trolox before the photosensitization. ${ }^{13,14)}$ Therefore, the leakage of potassium caused by the irradiation may also be due to the damage of RBC membranes by ROS. Although the use of an antioxidant could potentially minimize the leakage which may result from the irradiation, trials using antioxidants to prevent potassium leakage are rare. ${ }^{15)}$ Here we investigated the effect of antioxidants DPM, Trolox, human plasma or mannitol on the leakage of potassium ascribed to the irradiation of RBCs.

\section{MATERIALS AND METHODS}

Preparation of RBC Samples and Irradiation $\mathrm{RBC}$ in MAP solution (RC-MAP) were prepared according to the standard protocol of the Hokkaido Red Cross Blood Center. Briefly, after the centrifugation of donated human blood $(400 \mathrm{ml})$ at $3000 \mathrm{~g}$ for $8 \mathrm{~min}$, the supernatant was removed and $92 \mathrm{ml}$ of MAP solution (1.34 g D-mannitol, $0.013 \mathrm{~g}$ adenin, $\quad 0.086 \mathrm{~g}$ sodium dihydrogenphosphate, $0.138 \mathrm{~g}$ sodium citrate dihydrate, $0.018 \mathrm{~g}$ citric acid, $0.663 \mathrm{~g}$ D-glucose, and $0.457 \mathrm{~g}$ sodium chloride) was added (hematocrit: approximately $60 \%$ ). RC-MAP stored for $7 \mathrm{~d}$ at $4{ }^{\circ} \mathrm{C}$ was mixed with the same volume of MAP solution containing DPM, Trolox, human plasma or mannitol. RBC preparations $(30 \mathrm{ml})$ in plastic tubes $(50 \mathrm{ml}$ centrifuge tube; IWAKI Inc., Tokyo, Japan) were agitated for $2 \mathrm{~h}$ at room temperature and subsequently exposed to $30 \mathrm{~Gy}$ of gamma ray irradiation (irradiation time: $38 \mathrm{~min}$ ), using a $\mathrm{Cs}^{137}$ source (Gamma cell 40; Nordion International Inc., Kanata, Canada). After irradiation, the $\mathrm{RBC}$ preparations were stored in plastic tubes containing antioxidants at $4{ }^{\circ} \mathrm{C}$ for $7 \mathrm{~d}$.

Measurement of Potassium Concentration and Osmotic Pressure After the RBC preparations stored for $7 \mathrm{~d}$ at $4{ }^{\circ} \mathrm{C}$ 
after irradiation were centrifuged $(3000 \boldsymbol{g}, 10 \mathrm{~min})$, the potassium concentrations of the supernatants were measured. The concentration of potassium in the supernatant was measured using an instrument with an ion-specific electrode (644 $\mathrm{Na} / \mathrm{K} / \mathrm{Cl}$ Analyzer, Bayer Corporation, MA, U.S.A.). The osmotic pressure of MAP solutions was measured (Osmotic Pressure AUTO \& STAT, ARKRAY Inc, Kyoto, Japan) based on the depression of the melting point.

Chemicals DPM, Trolox, mannitol, and dimethyl sulfoxide (DMSO) were purchased from Sigma Chemical Company (St. Louis, MO, U.S.A.). DPM and Trolox were dissolved in DMSO as stock solutions. D-Mannitol, adenin, sodium dihydrogenphosphate, sodium citrate dihydrate, citric acid, D-glucose, and sodium chloride were purchased from Kanto Chemical Co. Inc. (Tokyo, Japan). Human plasma, which was obtained from healthy donors, was kept at $-80^{\circ} \mathrm{C}$ prior to use.

Statistical Analysis An ANOVA with Bonferroni correction was used to analyze the effect of the antioxidants on the irradiation-induced potassium leakage. "Potassium increase" refers to the difference between the external potassium concentration of the RBS preparations before irradiation and the concentration $7 \mathrm{~d}$ after irradiation. A value of $p<0.01$ was considered significant.

\section{RESULTS AND DISCUSSION}

The RBC preparations (hematocrit; 30\%) containing DPM, Trolox, human plasma or mannitol were irradiated at $30 \mathrm{~Gy}$ and subsequently stored at $4{ }^{\circ} \mathrm{C}$ for $7 \mathrm{~d}$. As shown in Figs. $1 \mathrm{~A}-\mathrm{C}$, the increase in the external potassium concentration of RBC preparations was markedly enhanced by the irradiation, which is consistent with a previous report. ${ }^{16)}$

DPM, which is widely used in the treatment of cardiovascular disease, binds noncovalently with high affinity $\left(K_{\mathrm{d}}=\right.$ $1.2 \mu \mathrm{M}$ ) to band 3, which is the most abundant protein on the RBC membrane with about a million copies per cell. ${ }^{14)}$ It has been reported that dipyridamole exerts an inhibitory effect on lipid peroxidation ${ }^{17)}$ and acts as a scavenger of superoxide anion, hydroxyl radical and peroxyl radical. ${ }^{18,19)}$ These properties make dipyridamole a candidate for an agent providing selective protection to RBCs against oxidative damage. Trolox, a water-soluble derivative of vitamin $\mathrm{E}$, is reported to bind efficiently to the RBCs membrane and is effective in quenching type I photoreactants (free radicals) that are produced in RBC by photosensitization. ${ }^{13)}$ It was reported that both of these antioxidants sufficiently inhibited the leakage of potassium from RBCs on photosensitization, which indicates that the leakage is due to the oxidative damage of RBC membranes by ROS produced from the photosensitizer. ${ }^{13,14)}$ In this study, the increase in extracellular potassium following irradiation was clarified to be significantly suppressed by the addition of DPM or Trolox (Figs. 1A, B). The leakage of potassium from the non-irradiated $\mathrm{RBC}$ preparations was unaffected by the antioxidants. Heat treatment at $37^{\circ} \mathrm{C}$, which enhances the fluidity of membrane phospholipids, ${ }^{20,21)}$ after irradiation was reported to suppress the leakage of potassium from RBCs. ${ }^{22}$ These findings indicate that ROS produced by the irradiation induced the increase in extracellular potassium through oxidative damage of the RBC membranes, similar to the case for photosensitization.

Whole blood contains not only cell components such as RBCs but also plasma. Human plasma is known to possess powerful antioxidants including vitamin $\mathrm{E}$, vitamin $\mathrm{C}$, the iron-free fraction of serum transferrin, and the copper-containing serum protein ceruloplasmin which can scavenge ROS generated by irradiation. ${ }^{23)}$ Therefore, human plasma is also expected to inhibit the leakage of potassium ascribed to the irradiation. After RC-MAP was mixed with the same volume of human plasma, the RBC preparation was agitated for $2 \mathrm{~h}$ at room temperature and subsequently irradiated. The leakage ascribed to the irradiaton was significantly suppressed by human plasma, which is consistent with the hypothesis that the leakage caused by irradiation is due to ROS (Fig. 1C).

Mannitol $(80 \mathrm{~mm})$, which is a constituent of MAP solution and used to inhibit the hemolysis of RBC during storage, is able to quench ROS such as the hydroxyl radical. ${ }^{24)}$ Therefore, mannitol is also expected to suppress the leakage of potassium from irradiated RBCs. We tried to clarify the effect of mannitol on the increase in potassium promoted by the irradiation. The increase in the external potassium concentration of the irradiated or non-irradiated RBCs was unexpectedly promoted in a manner dependent on the concentration of mannitol $(p<0.01)$ (Fig. 1D). The difference between the increase with irradation and without irradiation,
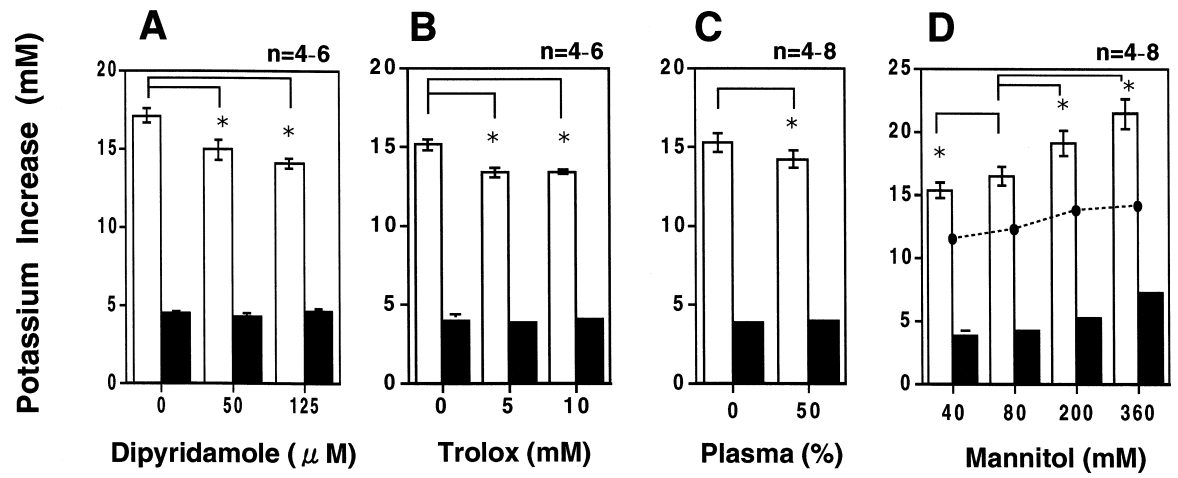

Fig. 1. The Effect of Antioxidants on the Leakage of Potassium Promoted by the Irradiation

The irradiated (white bar) or non-irradiated (black bar) RBC preparations were stored at $4{ }^{\circ} \mathrm{C}$ for $7 \mathrm{~d}$. The potassium increase means the difference between the external potassium concentration of RBC preparations before irradiation $(6.23 \pm 0.56 \mathrm{~mm}, n=4)$ and after $7 \mathrm{~d}$ storage. The closed circle shows the difference in the potassium increase between the irradiated RBCs (white bar) and non-irradiated RBCs (black bar), which is the leakage of potassium ascribed to the irradiation. Data shown are the mean \pm S.D. of four to eight experiments $(* p<0.01)$. 
Table 1. The Relation between Osmotic Pressure and Mannitiol Concentration of MAP Solutions

\begin{tabular}{lcccc}
\hline \hline Mannitol (mM) & 40 & 80 & 200 & 360 \\
\hline $\mathrm{mOsm} / \mathrm{kg} \mathrm{H}_{2} \mathrm{O}$ & $279 \pm 2.06$ & $320 \pm 1.41$ & $452 \pm 6.65$ & $625 \pm 14.0$ \\
\hline$n=4$ (mean \pm S.D.). & & & & \\
\end{tabular}

which is the increase ascribed to the irradiation, was also slightly enhanced dependent on the mannitol concentration (Fig. 1D, closed circle). It was reported that mannitol $(>100 \mathrm{~mm})$ decreased cell volume and reduced the filterability of RBCs through micropores, ${ }^{25,26)}$ which suggests structural changes of the RBC membrane. The osmotic pressure of MAP solution was increased in a manner dependent on the concentration of mannitol (Table 1). The structural change of $\mathrm{RBC}$ membranes at an elevated osmotic pressure may promote the leakage, which would be greater than the antioxidative suppression by mannitol. However, it is difficult to explain why the leakage of potassium ascribed to the irradiation was further enhanced dependent on the concentration of manner (Fig. 1D, closed circle). The RBC membrane may become extremely sensitive to irradiation on the addition of mannitol. Further investigation will be needed. In this study, mannitol $(80 \mathrm{~mm})$, which is used to inhibit hemolysis as a constituent of MAP solution, was shown to promote the leakage of potassium after irradiation. The amount of mannitol in MAP solution may have to be reconsidered.

In conclusion, the extent of irradiaiton-induced potassium leakage suppressed by DPM, Trolox or human plasma (10 $20 \%$ ) seems to be insufficient for the clinical use of these agents as additives in MAP solution, although antioxidants may be capable of suppressing irradiation-induced potassium leakage.

\section{REFERENCES}

1) Brubaker D. B., "Irradiation of Blood Components," ed. by Baldwin M. L., Jefferies L. C., American Association of Blood Banks,
Bethesda, 1992, pp. 1-30.

2) Von Fliedner V., Higby D. J., Kim U., Am. J. Med., 72, 951-961 (1982).

3) Leitman S. F., Holland P. V., Transfusion, 25, 293-304 (1985).

4) Valerius N. H., Johansen K. S., Nielsen O. S., Platz P., Rozenkvist J., Sorensen H., Scand. J. Haematol., 27, 9-18 (1981).

5) Sprent J., Anderson R. E., Miller J. F., Eur. J. Immunol., 4, 204-210 (1974).

6) Button L. N., DeWolf W. C., Newburger P. E., Jacobson M. S., Kevy S. V., Transfusion, 21, 419-426 (1981).

7) Brugnara C., Churchill W. H., Transfusion, 32, 246-252 (1992).

8) Kankura T., Nakamura W., Eto H., Nakao M., Int. J. Radiat. Biol. Relat. Stud. Phys. Chem. Med., 15, 125-136 (1969).

9) Brown K. A., Bissonnette B., MacDonald M., Poon A. O., Can. J. Anaesth., 34, 401-408 (1990).

10) Barnard D. R., Chapman R. G., Simmons M. A., Hathaway W. E., Transfusion, 20, 401-408 (1980).

11) Galligan B. R., Cairns R., Schifano J. V., Selbring A., Bernvil S. S., Transfusion, 29, 179-181 (1989).

12) Singh A., Singh H., Prog. Biophys. Mol. Biol., 39, 69-107 (1982).

13) Ben-Hur E., Rywkin S., Rosenthal I., Geacintov N. E., Horowitz B., Transfusion, 35, 401-406 (1995).

14) vanSteveninck J., Trannoy L. L., Besselink G. A., Dubbelman T. M., Brand A., deKorte D., Verhoeven A. J., Lagerberg J. W., Transfusion, 40, 1330-1336 (2000).

15) Sharifi S., Dzik W. H., Sadrzadeh S. M. H., Transfus. Med., 10, 125130 (2000).

16) Chicha I., Suzuki Y., Tateishi N., Shiba M., Muraoka M., Tadokoro K., Maeda N., Vox Sang., 79, 75-82 (2000).

17) Nepomuceno M. F., Alonso A., Pereira-da-Silva L., Tabak M., Free Radic. Biol. Med., 23, 1046-1054 (1997).

18) Iuliano L., Pedersen J. Z., Rotilio G., Ferro D., Violi F., Free Radic. Biol. Med., 18, 239-247 (1995).

19) Iuliano L., Pratico D., Ghisell A., Bonavita M. S., Violi F., Lipids, 27, 349-353 (1992).

20) Inbar M., Shinitzky M., Sachs L., FEBS Lett., 38, 268-270 (1974).

21) Shinitzky M., Inbar M., J. Mol. Biol., 85, 603-615 (1974).

22) Anderson G., Mintz P. D., Ann. Clin. Lab. Sci., 24, 339-345 (1994).

23) Halliwell B., Gutteridge J. M., Arch. Biochem. Biophys., 280, $1-8$ (1990).

24) Goldstein S., Czapski G., Int. J. Radiat. Biol. Relat. Stud. Phys. Chem. Med., 46, 725-729 (1984).

25) Sasakawa S., Shibata M., Mura T., Nakajima T., Suzuki Y., Maeda N., Jpn. J. Transfus. Med., 37, 398- 403 (1991).

26) Nakao M., Nakao T., Komatsu Y., Sano K., Sasakawa S., Biomed. Biochim. Acta, 42, 527-535 (1983). 